but there are no adequate comparators. CBZ and PHT may precipitate or aggravate generalized-onset tonic-clonic seizures (class IV suggestive evidence).

In children with absence seizures, 3 AEDs (VPA, ESM, and LTG) had new class I or class II evidence of efficacy; LEV had additional class III evidence of efficacy. ESM and VPA are adequate comparators and are established (level A); LTG is possibly (level C) effective. GBP is established as ineffective (level F); AEDs that may precipitate or aggravate absence seizures (class IV scattered reports) include CBZ, OXC, PB, PHT, TGB, and VGB. LEV failed class III placebo-controlled trial and efficacy is undetermined.

In children with BECTS, CBZ and VPA are possibly (level C) and GBP, LEV, $\mathrm{OXC}$, and STM are potentially (level D) effective. For juvenile myoclonic epilepsy, TPM and VPA are potentially (level D) effective; CBZ, GBP, OXC, PHT, TGB, and VGB may precipitate or aggravate absence, myoclonic, and sometimes, generalized tonic-clonic seizures. LTG exacerbated seizures in JME (level F) in one report. (Glauser T, BenMenachem E, Bourgeois B, et al. Updated ILAE evidence review of antiepileptic drug efficacy and effectiveness as initial monotherapy for epileptic seizures and syndromes. Epilepsia 2013 Mar;54(3):551-63). (Response: Tracy A Glauser, Comprehensive Epilepsy Center, Division of Neurology, Cincinnati Children's Hospital, OH 45229. Email: tracy.glauser@cchmc.org).

COMMENT. The authors stress that their report is a working framework and not a mandatory rulebook. Multicenter studies and trial designs are needed to determine the efficacy of new AEDs compared to the old, but the most appropriate AED for a specific patient is decided by the judgment and expertise of the individual physician. Management of epilepsy remains partly an art as much as a science, especially when polytherapy is involved and overused.

\title{
ENCEPHALOPATHIES
}

\section{POSTERIOR REVERSIBLE ENCEPHALOPATHY SYNDROME}

Investigators at University of Milan, Italy, report a 6-year-old boy who presented with left-sided weakness followed by status epilepticus, left-sided tonic-clonic seizures with secondary generalization, without fever. The seizure was preceded by an acute gastroenteritis in the week prior to admission. His blood pressure was $139-112 \mathrm{~mm} / \mathrm{Hg}$ $\left(>95^{\text {th }}\right.$ percentile). Control of status by iv phenobarbital was accompanied by resolution of hypertension. Neurological examination revealed hypotonia and hyporeflexia of the left side. Blood and CSF examinations were normal, including PCR for Enterovirus. MRI showed bilateral asymmetric low intensities in the white and gray matter of posterior parietal and occipital lobes, affecting the right hemisphere predominantly. EEG showed an encephalopathic pattern in posterior regions with no epileptiform discharges. The patient recovered completely within the following 6 days, and follow-up exams in 3 and 12 months were normal, including neurological, EEG, MRI, and blood pressure, and with no recurrence of seizures. (Mameli C, Dilillo D, Spiri D, Cerini C, Fasan S, Zuccotti GV. Status epilepticus as manifestation of posterior reversible encephalopathy syndrome in a healthy child. Pediatr Neurol 2013 May;48(5):418-20). (Response: Dr Mameli, 
Department of Pediatrics, L Sacco Hospital, University of Milan, 20157 Milano, Italy. Email: mameli.chiara@hsacco.it).

COMMENT. Posterior reversible encephalopathy syndrome (PRES), first described in 1996 (Hinchey J, et al. N Engl J Med 1996 Feb 22;334(8):494-500), is characterized clinically by headache, altered awareness, visual disturbance, and seizures, and radiologically by transient posterior lesions in subcortical white matter. PRES is associated with a rapid rise in blood pressure that may underlie the encephalopathy. The pathophysiology of PRES is not completely understood, but predisposing conditions include renal and hemato-oncologic diseases and use of chemotherapeutic immunosuppressive drugs. (Siebert E, et al. Eur J Paediatr Neurol 2013 Mar;17(2):16975 [Cited by Mameli]). Other conditions reported in association with PRES are organ and bone marrow transplantation, autoimmune disease, Guillain-Barre syndrome, sickle cell anemia, hemolytic-uremic syndrome, and iv immunoglobulin administration.

\section{SLC19A3 EARLY-INFANTILE, LETHAL ENCEPHALOPATHY}

Investigators from VU Medical Centre, Amsterdam, The Netherlands, identified seven patients with severe encephalopathy who shared a previously undescribed MRI pattern with cystic degeneration of the white matter and progressive cerebral, cerebellar and brainstem atrophy. All patients showed rapid deterioration of brain function soon after birth, followed by respiratory failure and death. Whole-exome sequencing revealed pathogenic, heterozygous missense mutations in the SLC19A3 gene, encoding the second thiamine transporter. Pathology of brain tissue demonstrates cerebral atrophy and lesions similar to Leigh's syndrome. This new, severe, lethal phenotype broadens the phenotypic spectrum of SLC19A3 mutations and is recognized by the associated MRI pattern of brain degeneration. (Kevelam SH, Bugiani M, Salomons GS, et al. Exome sequencing reveals mutated SLC19A3 in patients with an early-infantile, lethal encephalopathy. Brain 2013 May;136(Pt 5):1534-43). (Response: Marjo S van der Knaap, Department of Child Neurology, VU Medical Centre, de Boelelaan 1117, 1081 HV Amsterdam, The Netherlands. E-mail: ms.vanderknaap@vumc.nl).

COMMENT. MRI pattern of initial swelling with T-hyperintensities followed by rapid degeneration and brain atrophy allows early diagnosis of a rapidly progressive infantile encephalopathy caused by SLC19A3 mutations.

\section{MIGRATING PARTIAL SEIZURES OF INFANCY}

A national surveillance study in conjunction with the British Paediatric Neurology Unit was undertaken to further define the clinical, pathological and molecular genetic features of migrating partial seizures of infancy (MPSI), a rare early infantile epileptic encephalopathy with poor prognosis. In 14 patients reported during the 2 year study period, MPSI was associated with an expanded spectrum of clinical features including gut dysmotility and movement disorder, EEG features including hypsarrhythmia with infantile spasms and burst suppression, and novel brain imaging including delayed myelination, white matter hyperintensity and in one patient at autopsy, putaminal 